\title{
Using interaction-based phenotyping to assess the behavioral and neural mechanisms of transdiagnostic social impairments in psychiatry
}

\author{
Leonhard Schilbach ${ }^{1}$
}

Published online: 13 March 2019

๑) Springer-Verlag GmbH Germany, part of Springer Nature 2019

The idea that we should think of psychiatric disorders as "brain disorders" has been around for over 150 years. Over the decades, we have seen an impressive refinement of the methods and tools that have been put to use, but until today and in contrast to other branches of medicine such as oncology that have been highly successful in personalizing treatments we do not have any specific and reliable brain-based biomarkers available in psychiatry. This is all the more troubling as the global burden of mental health conditions is on the rise: In light of the dramatic numbers and a "monumental loss of human capabilities" resulting from mental ill health, the Lancet Commission on global mental health has recently spoken of a "global health crisis" and a "collective failure to respond to this". Furthermore, the commission recognized the complex nature of psychiatric disorders and mental health by characterizing the latter as "the unique product of social and environmental influences $[\ldots]$ interacting with genetic, neurodevelopmental and psychological processes and affecting biological pathways in the brain". This characterization is reflected by the kind of research that has and is being conducted in psychiatry and the clinical neurosciences. Indeed, this research has focused on the genetic contributions to psychiatric disorders, but more recently also on the epigenetic factors, i.e., gene $\times$ environment interactions, which are relevant in shaping mental ill health [1]. In spite of the important advances and new insights that have been gained as a result of focusing more strongly on the interplay between social and biological factors [2], our understanding of how social factors influence the "social brain' and how this could inform individual diagnosis and

Leonhard Schilbach

leonhard_schilbach@psych.mpg.de

1 Outpatient and Day Clinic for Disorders of Social Interaction and Independent Max Planck Research Group for Social Neuroscience, Max-Planck Institute of Psychiatry, Kraepelinstr. 2-10, 80804 Munich, Germany treatment is still limited. Furthermore, social impairments are ubiquitous across different psychiatric disorders, which (amongst other aspects) has led to the suggestion that we can describe them as disorders of social interaction, but also that we should focus on whether disordered social interactions are the result of disorder-general or disorder-specific mechanisms [3].

In light of the above-described difficulties of establishing brain-based biomarkers for psychiatry, an important recent suggestion has been the idea that we could use 'digital phenotyping', i.e., sensor- or smartphone-based ways to quantitatively assess behavior and use this information to detect and treat mental ill health [4]. There is much to like about this proposal, because it suggests measuring behavior where it really matters, in everyday life, which promises to help focus on therapeutically relevant phenomena. By doing so, the proposal parallels recent developments in the field of social neuroscience, where a focus on more ecologically valid social situations and, thanks to methodological advances, the study of real-time social interactions has helped to shed new light on the neural mechanisms of relatively unconstrained social encounters and how they can be disturbed in the case of mental illness [5]. What is still missing, however, are unobtrusive motion tracking tools that unlike smartphones can assess the behavioral dynamics that take place in real-life social interaction at the dyadic (or even multi-person) level. Next to digital phenotyping, psychiatry could, therefore, make use of interaction-based phenotyping to quantify the level of reciprocity of social behavior between two persons, i.e., how person A's behavior relates to that of person B.

Rather than relying on individual read outs of behavior as in the past decades, this approach could help psychiatry to establish a quantitative approach to analysing interactionbased phenomena, which so far have been exclusively left to clinical intuition (as the praecox feeling in schizophrenia or a lack of social presence in autism). Interaction-based 
phenotyping could be made compatible with point of care situation in psychiatric clinics and could deliver a multitude of additional data to generate an observer-independent social interaction 'footprint' of an individual that could give us completely new insights about the social repertoire of a patient [6]. Such measures might be helpful to quantitatively assess autistic, but also other interactionally relevant tendencies and traits of a person, whose assessment is of high clinical importance to understand heterogeneity in affective and anxiety disorders and has been targeted by recent developments in psychotherapy. Furthermore, it has been shown that interaction-based measures such as interpersonal synchrony may have predictive value for treatment outcome [7]. It is also conceivable that interaction-based phenotyping could be developed further to support psychotherapy training as it may make it possible to revisit and analyze key moments of the social interaction based on objective read outs. It is tempting to speculate that interaction-based assessments could help to scientifically assess the likelihood that a therapist and a patient will 'click' and work well together. In addition, interaction-based phenotyping could help to explain why, for instance, social interactions between persons with autism tend to work better than dyads where one person is autistic and the other is not, which has been described as the 'social interaction mismatch hypothesis' of autism [8].

Importantly new interaction-based measures, e.g., the level of interpersonal synchrony of behavior in patients with autism with an interviewer, could also be related to measures from established, well-controlled experimental paradigms such as motor-generation task used in the important longitudinal study by [9] to investigate response inhibition in boys with. Systematically investigating the relationship of more and less ecologically valid and more and less well-controlled tasks could be informative in autism research where we find that patients follow instruction in so-called social tasks so well that group differences cannot always be found [10], but also in all other areas of psychiatry where social impairments exist. Also, such an assessment could be particularly relevant to further understand the most interesting longitudinal changes that Weiss et al. report in their study [9] and their possible interplay with other factors.

Finally, interaction-based phenotyping also promises to make social neuroscience better and more relevant for psychiatry: by allowing to quantitatively assess social interactions, this method might serve as a 'calibration device', because it may allow us to isolate social phenomena in ecologically valid situations that have so far escaped scientific investigations. Such phenomena could then be studied by means of established techniques such as functional neuroimaging and experimental tasks, which are informed by data from interaction-based phenotyping. Such a cross-fertilization of methods might yield completely new insights into the relevant neural mechanisms and as such could improve our understanding of psychiatric disorders as disorders of the (social) brain, but it would also strengthen an integrative view that also sees psychiatric disorders as disorders of social interaction and focuses on how the experiences that we make with others shape our mental health.

\section{References}

1. Binder E (2017) Understanding gene $x$ early adversity interactions-possibilities for insights into the biology of psychiatric disorders. Eur Arch Psychiatry Clin Neurosci 267:183-185. https ://doi.org/10.1007/s00406-017-0775-0

2. Haddad L, Schäfer A, Streit F et al (2014) Brain structure correlates of urban upbringing, an environmental risk factor for schizophrenia. Schizophr Bull 41(1):115-122

3. Schilbach L (2016) Towards a second-person neuropsychiatry. Philos Trans R Soc Lond B 371(1686):20150081

4. Insel T (2017) Digital phenotyping. JAMA 318(13):1215-1216

5. Redcay E, Schilbach L (2019) Using second-person neuroscience to elucidate the mechanisms of social interaction. Nat Rev Neurosci (in press)

6. Leong V, Schilbach L (2019) The promise of two-person neuroscience for developmental psychiatry: Using interaction-based sociometrics to identify disorders of social interaction. Br J Psychiatry. https://doi.org/10.31234/osf.io/9upxf

7. Koole SL, Tschacher W (2016) Synchrony in psychotherapy: a review and an integrative framework for the therapeutic alliance. Front Psychol 7:862

8. Bolis D et al (2017) Beyond autism: introducing the dialectical misattunement hypothesis and Bayesian account of intersubjectivity. Psychopathology 50(6):355-372

9. Weiss et al (2018) Age-moderating effect in prepotent response inhibition in boys with Asperger syndrome: a 2.5 years longitudinal study. Eur Arch Psychiatry Clin Neurosci. https://doi. org/10.1007/s00406-018-0915-1

10. Cusack JP, Williams JH, Neri P (2015) Action perception is intact in autism spectrum disorder. J Neurosci 35(5):1849-1857 